$\underline{\text { Review Article }}$

\title{
PROSPECTIVE AND RETROSPECTIVE ANIMAL MODEL USED IN THE PHARMACOLOGICAL SCREENING OF ANTI-CANCER DRUG
}

\author{
SHAMIMA NASREEN AHMED*, BISWAJIT DAS ${ }^{\text {, }}$ JASHABIR CHAKRABORTYb \\ *Girijananda Chowdhury Institute of Pharmaceutical Science, Guwahati-17, aPharmaceutical Biotechnology, Girijananda Chowdhury Institute \\ of Pharmaceutical Science, Guwahati-17, bPharmacology, Girijananda Chowdhury Institute of Pharmaceutical Science, Guwahati-17 \\ Email: ahmedshamima11@gmail.com
}

Received: 21 Apr 2018, Revised and Accepted: 10 Jun 2018

\begin{abstract}
Cancer is a disease characterized by uncontrolled proliferation of cells that have transformed from the normal cells of the body. The widely used cancer drugs suffers from the drawback of high toxicity not within the reach of a common man. This urgently necessitating the screening of these compounds. This review focuses on the major contributions of preclinical screening models to anticancer drug development over the years till recent times, from the empirical drug screening of cytotoxic agents against uncharacterized tumor models to the target-orientated drug screening of agents with defined mechanisms of action, a general transition has been observed. The newer approaches to anticancer drug development involve the molecular characterization of models along with an appreciation of the pharmacodynamics and pharmacokinetic properties of compounds [e. g., the US National Cancer Institute (NCI) in vitro 60-cell line panel, hollow fibre assay, and s. c. xenograft]. In vivo tumor models including orthotopic, metastatic, and genetically engineered mouse models are also reviewed. The preclinical screening efforts of the European are also included. In 2015 with the rapid development of cancer modeling in zebrafish, great opportunities exist for chemical screens to find anticancer drug since 1970 the European Organisation for Research and Treatment of Cancer and Cancer Research UK, have been collaborating with the NCI in the acquisition and screening of compounds.
\end{abstract}

Keywords: Proliferation, Preclinical, Cytotoxic agents, Xenograft, Zebrafish

(C) 2018 The Authors. Published by Innovare Academic Sciences Pvt Ltd. This is an open access article under the CC BY license (http://creativecommons.org/licenses/by/4.0/] DOI: http://dx.doi.org/10.22159/ijcpr.2018v10i4.28472

\section{INTRODUCTION}

Cancer, the uncontrolled growth of cells, is a major cause of death throughout the world, in the recent years, it killed $\sim 7900000$ people worldwide, a value that represents $\sim 13 \%$ of death. The cancer cells can invade the adjacent and distant tissue via the circulation. In advanced stages, one of the causes of treatment failure is the development of resistance to anticancer drugs. Cancer is a multifactorial disease the biology of which is still not yet fully understood. However, the induction of proto-oncogenes and inhibition of tumour suppressor genes has been implicated in the pathogenesis of cancer. Angiogenesis plays an important role in the pathogenesis of cancer and is a common target for the chemotherapeutic agents. For such a dreadful disease, anticancer drugs have been developed from a variety of sources ranging from natural products to synthetic molecules. The challenging task at this moment is to identify the quick and novel methods that can identify and develop molecules, which can be of therapeutic value in human cancers. This urgently necessitates screening of a large number of compounds. For this purpose different models are employed for the systematic screening of anticancer drugs. In this review, screening of anticancer drugs are described with a focus on their strength and limitations.

\section{Tumor models (1955-1985)}

In 1955, the National Cancer Institute (NCI) began a large-scale anticancer drug screening program testing agents against a panel of three mouse tumor models: sarcoma 180, L1210 leukemia, and carcinoma 755. In 1965, screening was limited to the use of only the L1210 and Walker 256 carcinosarcoma murine models. By 1968, synthetic agents were screened in L1210 alone, whereas natural plant products were screened in L1210 and P388 leukemias. 1972, B16 melanoma and the Lewis lung carcinoma mouse models were introduced. During the first $25 \mathrm{y}$, clinical response of human leukemias and lymphomas improved, whereas treatment response was less promising for most human solid tumors [1]. As a consequence, in 1976 the Division of Cancer Treatment at the NCI introduced a new tumor panel incorporating transplantable solid "human" tumor models that were representative of the major histologic types of cancer prevalent in the United States at the time. This development followed the revolutionary discovery of the nude athymic (nu/nu) mouse and the successful growth of human tumor xenografts. The panel consisted of matched animal and human tumors of the breast (CD8F1/MX-1), colon (colon 38/CX-1), and lung (Lewis/lX-1), along with L1210 leukemia and B16 melanoma syngeneic models. Syngeneic models involved inoculation i. p., s. c., or i. v., whereas human tumor xenografts were grown under the sub renal capsule. The subrenal capsule assay uses small tumor fragments growing under therenal capsule of athymic mice and normal immunocompetent mice. Although the subrenal capsule assay was labour-intensive, it provided a rapid means of evaluating new agents against human tumor xenografts at a time when longerterms. c. assays were not feasible. The subrenal capsule assay has shown the good predictive value of clinical response with an overall valuable assay of $90 \%$. In vivo "pre-screen" using the P388 model was also used.

In 1976-1982, the mouse-human tumor panel identified antitumor agents (e. g., taxol) that would have been missed by the L1210 model alone. Approximately $30 \%$ of compounds found to be active in at least one human tumor xenografts were missed by syngeneic models. Therefore, the mouse-human tumor panel successfully achieved the goal of providing new agents for a clinical trial. In 1982 the NCI employed a new strategy involving a sequential process of 'progressive selection'. Compounds were first subjected to the P388 prescreen model, and then a standard panel including tumor models known to produce a relatively high-yield of active compounds (MX1, B16, andL1210). A new model, the M5076 sarcoma as well as advanced and multidrug-resistant tumor models, were incorporated in this standard panel. Agents found to be active in the standard tumor panel were subjected to secondary screening [2]. The selection of secondary tumor models was "drug-orientated." Agents were subjected to specific tumor models based on the known properties of each individual compound and previous experience in the standard tumor panel. At this time, several retrospective preclinical-clinical correlation studies were reviewed, but no 
apparent positive correlation between preclinical and clinical efficacy based on tumor type was found. It was suggested that the lack of histologically based correlations may be a consequence of experimental design which limited tumors to one mouse and one human correlation for each of the three major types. It was also suggested that a "model system" composed of several tumors of the same type might, on the basis of percentage responders, predict for a reasonable clinical response rate against similar type tumors.

\section{Human tumor stem cell assay/ $\$ clonogenic assay}

The Human Tumor Stem Cell Concept The study of cell proliferation was advanced by adapting murine [1] and human [2] hematopoietic cells in the soft agar system and developing a similar methodology for solid human tumors. Consequently, a cell renewal hierarchy concept was postulated proposing that the neoplastic population constitutes a spectrum of cells ranging from those with the capacity to renew the entire cell population, including themselves, to those with a limited number of potential divisions. Cells capable of selfrenewal and clonal expansion, called stem cells, regulate the growth of the primary tumor and its metastatic growth. The other subpopulations of cells in this schema consist of transitional or "intermediate" cells with the limited \proliferative ability and "end" with nonproliferative differentiated properties

The applicability of the HTSC assay for drug screening purposes in terms of feasibility, validity, and potential to identify new antitumor agents was investigated [3]. The testing of established standard chemotherapeutic agents in this pilot study revealed that most agents were found to be active with the exception of those requiring systemic activation. Clinically ineffective agents were confirmed to be true negatives with $97 \%$ accuracy. Other groups also showed the potential use of the assay in predicting clinical activity. Typically, an assay was shown to predict drug resistance with $90 \%$ accuracy and clinical drug sensitivity with between $40 \%$ and $70 \%$ accuracy. Additionally, of 79 compounds found previously to be inactive using the P388 prescreen, 14 were active in the HTSC assay. Reproducibility of survival values within assays and between laboratories was also revealed. However, several limitations prevented the use of this assay for large-scale screening, the main criticism being that many tumor types have a low plating efficiency. Subsequently, only breast, colorectal, kidney, lung, melanomas, and ovarian tumors produce a sufficient yield of evaluable assays. Hence, the number of patients for whom treatment may be chosen by clonogenic assays was frequently $<50 \%$, although recently the growth rates of primary tumor tissues in the HTSC assay has significantly improved (70-80\%). Additional problems encountered include labor intensity, automation, and difficulty in attaining a single cell suspension from human solid tumors. Clonogenic assays also include sources of substantial error, and quantification of data, cell survival curves, and colony size are often criticized. To date, there are no phase III clinical studies of individualized therapy demonstrating a significant increase in survival compared with empirically determined standard treatment, therefore the clonogenic assay has not found a practical established role in the individualization of patient therapy [4]. Clonogenic assays are still widely used as a secondary screen by independent researchers. At the Institute for Experimental Oncology in Freiburg, an in vitro/in vivo testing procedure is employed using target-defined tumor models. Patients' tumors are tested directly in the in vitro clonogenic assay, or after being established as a permanent xenograft model. Agents are tested using both an empirical and target-orientated screening strategy. In addition to the routine endpoint of the clonogenic assay (inhibition of colony growth), pharmacodynamic assays are employed to determine compound activity.

\section{Human tumor in vitro cell line screen, 1985 to the present day}

Since April 1990, DTP has used the human tumor cell line in vitro screen as its primary assay. The screen is currently composed of 59 human tumor cell lines, representing leukemia, melanoma, and cancers of the lung, colon, brain, ovary, breast, prostate, and kidney. These cell lines were selected partly on pragmatic terms: those selected behaved best under assay conditions. The screen was designed so that for each compound tested, both the absolute and the relative sensitivities of individual cell lines were reproducible to the extent that a characteristic profile or fingerprint of cellular response was generated.

Although the particular inhibitory response of a single cell line might be relatively uninformative, the pattern of response of the cell lines as a group can be used to rank a compound according to the likelihood of sharing common mechanisms. The COMPARE algorithm qualifies this pattern and searches an inventory of screened agents to compile a list of the compounds that have the most similar patterns of cellular sensitivity and resistance.

In 1985, the hypothesis was put forward that the human tumor cell line screen could discover cell type-specific agents with clinical activity against solid tumors. The emerging reality is that correlation of in vitro histology to clinical activity is poor, but the pattern of cellular sensitivity and resistance of the cell lines to the drug correlates with molecular target expression. The screen is now used as a cytotoxic assay but also to identify compounds with activity against cells that may relate to a particular molecular target profile

In 1999 an in vitro prescreen was introduced whereby compounds were screened in a three-cell line panel using three highly sensitive cell lines, MCF-7 (breast carcinoma), NCI-H460 (lung carcinoma), and SF-268 (glioma). The rationale for this prescreen was the observation that $85 \%$ of compounds screened in the past had shown no evidence of antiproliferative activity, and a three-line prescreen was shown to efficiently remove many of the inactive compounds from unnecessary and costly full-scale evaluation in the 60-line panel.

The present human tumor cell line in vitro screen is technically simple, relatively fast, cheap, reproducible, and provides valuable indicative data of mechanistic activity and target interaction. Yet it is not without its limitations. In vitro methods are susceptible to false-positive and false-negative results. It is also clear that factors other than the inherent chemo sensitivity of tumor cells significantly influence the outcome of chemotherapy in vivo (e. g., pharmacokinetics, tumor microregions $/ \mathrm{pH}$, and $\mathrm{p02}$ ). Such factors are not represented by the in vitro 60-cell line screening assay, yet it is appreciated that this assay was designed only to select compounds for a secondary, more comprehensive, in vivo testing. The original intention of the NCI/DTP was to produce a high-throughput in vitro screen that would be sufficiently discriminatory to ensure that only a relatively small number of compounds would be selected for further evaluation in human tumor xenograft models. This has not been the case and subsequently, the in vivo HFA was implemented in 1995 in attempt to prioritize compounds for secondary xenograftscreening and help reduce the large number compounds that were forming a bottleneck for entry into secondary xenograft testing.

\section{The hollow fiber assay}

The HF assay was developed by Hollingshead et al. at the NCI and is composed of 2-cm tubes filled with tumor cell lines. These fibers are implanted into mice at two sites (intraperitoneal and subcutaneous). The fibers are removed after 4-6 d in the animal and processed in vitro for quantification of tumor cell growth. By determining net cell kill, one can examine whether drugs administered via different routes are bioavailable and can reach the tumor sites. TheHFA assesses the pharmacologic capacity of compounds to reach two physiologic compartments within the nude mouse and shows a practical means of quantifying viable tumor cell mass [5].

The in vivo hollow fibre assay was developed at the NCI to help bridge the gap between the in vitro cell-based assays and human xenograft models in immuno-deficient mice. The goal was to develop an intermediate assay that could better predict which compounds found active in the 60-cell line panel would be active in subsequent xenograft models. This was necessary due to the high cost of the traditional xenograft assay in terms of a number of animals required, time for assay completion, and financial commitment necessary.

For the standard hollow fibre assay (HFA), the high and low dose levels are determined using the MTD as determined above using the formula below.

High dose $=[$ MTD $\times 1.5] / 4$ 


\section{Low dose $=0.67 \times$ high dose}

The standard vehicle used for both acute toxicity testing and HFA is $10 \%$ DMSO in saline $/ 0.05 \%$ Tween 80 . Primary Anti-cancer Drug Screening Activities.

\section{Xenografts models}

The review of $\mathrm{NCI}$ in vitro and in vivo screening efforts based on the 60 human cell line panel and xenograft testing in the 1990s has recently been published. The methods of the NCI procedures were mainly empirical during this time period and disease rather than target based. Data were available on 39 agents with both xenograft data and Phase II trial results. The analysts found that histology of a particular preclinical model showing in vivo activity did not correlate with activity in the same human cancer histology. However, drugs with in vivo activity in a third of the tested xenograft models did correlate with ultimate activity in some Phase II trials. This and the fact that none of the currently registered anticancer drugs was devoid of activity in preclinical tumor models, but showed activity in the clinic, led to the conclusion that activity in in vivo models of compounds demonstrating in vitro activity remains desirable.

The hollow fibre assay has proven a valuable interface for selecting development candidates from large pools of compounds with in vitro antiproliferative activity for expensive and time-consuming subcutaneous xenograft testing.

\section{a) In vitro models}

In vitro models to define the mechanisms of action of a given compound Once a compound has demonstrated robust cytotoxic activity against a panel of human cancer cell lines and deserves further investigation in in vivo models, it is important to clarify its mechanism of action and to identify its exact molecular targets. An example of the importance of the in vitro studies aimed at clarifying the mechanism of action of a given drug is represented by PARP inhibitors. The initial panel of cell lines incorporated a total of 60 cell lines representing nine distinct tumour types (leukaemia, colon, lung, CSN, renal, melanoma, ovarian, breast and prostate cancer). To date, more than 85,000 compounds have been screened against this in vitro panel of the short-term assay. Compounds are tested over a 5 - $\log$ concentration range against each of the 60 cell lines for their ability to inhibit the growth of, or to kill, the cells in a 2-day assay generating 60 dose-response curves.

\section{b) In vivo models}

The available in vivo models used to select compounds for further clinical development will be herein briefly summarised.

\section{Murine tumours}

P388 and L1210 leukaemia cell lines have been used for many years as the major preclinical models to screen new compounds. Therefore, in the early 1970s, the B16 melanoma and Lewis lung carcinoma were also incorporated to try to identify compounds potentially active in human solid tumours. These tumours also induced the formation of lung metastases and thus were of potential use for investigating both the antitumor and the anti-metastatic activity of new compounds.

\section{Genetically engineered cancer models (GEM)}

Over the past 20 y GEM models have been instrumental not only in our understanding of the molecular pathways responsible for the initiation and progression of tumours but also because they have highlighted the importance of specific oncogenes and tumour suppressor genes in carcinogenesis. As GEM models have been shown to partially recapitulate the genetic/molecular changes occurring in human tumours, the challenge now is to use these models to test novel anticancer therapies in an attempt to better select clinically effective compounds.

GEM models have increased our understanding of the molecular pathways responsible for the initiation and progression of human cancer, and have highlighted the importance of specific oncogenes and tumor suppressor genes (TSG) in particular types of cancer. GEM models possess well-validated molecular/genetic characteristics (e. g., gene mutations) which ultimately facilitate the rational design of small molecule therapeutics. The main aim of GEM models is to recapitulate genetic/molecular changes in human cancer and use these to test novel anticancer therapeutics in an attempt to accurately predict clinical response. The first strains of genetically engineered mice predisposed to cancer were transgenic mouse models whereby cellular/viral oncogenes were introduced to the mouse germ line. One of the first transgenic cancer models involved the constitutive expression of the c-myconcogene under the control of the mouse mammary tumor virus promoter leading to the development of mammary tumors.

\section{Orthotopic and metastasis tumor models}

As described previously, compounds are usually screened against a panel of poorly characterized human tumor xenografts implanted $s$. c. in nude mice. S. c. tumor models do not represent the primary site of common human cancers or sites of metastasis. It has been suggested that the disparity between preclinical and clinical activity is related to the treatment of advanced metastatic disease in the clinic, whereas conventional. c. xenograft models do not represent advanced metastatic diseasenor is the orthotopic site represented [6]. Orthotopic transplantation models attempt to mimic the morphology and growth \ characteristics of clinical disease [7-11] and are thought to represent a more clinically relevant tumor model with respect to the tumor site and metastasis. One of the most obvious advantages of orthotopic systems is that attempts to target processes involved in local invasion (e. g., angiogenesis) can be carried out in a more clinically relevant site. Several other models are also used to assess the antiangiogenic properties of novel agents (e. g., corneal micropocket assay; ref. 12). Since the early studies showing orthotopic transplantation of colon tumors and metastasis to the liver, tumor material has been grown orthotopically in mice at most common sites of human cancer. Whether preclinical models representative of clinical disease (e. g. orthotopic/metastatic models) should be employed as a replacement for traditional s. c. nonmetastatic xenografts [10] is an interesting question.

The characterization of metastasis models may be evaluated more easily with the use of noninvasivemicroimaging research tools Recent technological advances have had a major impact on research using orthotopic models for studying the process of cancer metastasis. Magnetic resonance imaging and positron emission tomography are being used to visualize tumor and metastasis progression. Reporter genes with specific fluorescence properties have been developed including the stable green fluorescent protein, galactosidaselac Z gene, and the luciferase gene. Although such technology is not widely used, it has permitted the visualization of tumor growth using ethically more acceptable noninvasive techniques and has reduced the numbers of animals required for orthotopic and metastasis studies.

\section{Autochthonous models}

Autochthonous tumors include spontaneously occurring tumors and induced tumor growth (e. g. by chemical, viral, or physical carcinogens). It is thought that autochthonous tumors may mimic human tumors more closely than transplanted tumors (i.e., s. c./orthotopic). Advantageous properties include orthotopical growth, tumor histology devoid of changes introduced by transplantation, and a route of metastasis through lymph and blood vessels that surrounded early tumor growth [13].

Despite such properties, the use of autochthonous tumor models has not been widespread due to several limitations. A large variability in take rate and growth exists, a large number of animals needed, time frames of several months to years exist for a single experiment due to long tumor latencies as opposed to weeks in transplanted xenograft models, and lack of spontaneous metastasis. Autochthonous models are used occasionally as tools for advanced or phase II screening [13], but more recently in this "post-genome era" autochthonous models have largely been replaced by genetically engineered mouse (GEM) models.

\section{Genetically engineered cancer models}

Over the past $20 \mathrm{y}$, GEM models have made a significant contribution to the field of cancer research. GEM models have increased our 
understanding of the molecular pathways responsible for the initiation and progression of human cancer, and have highlighted the importance of specific oncogenes and tumor suppressor genes (TSG) in particular types of cancer.

GEM models possess well-validated molecular/genetic characteristics (e. g., gene mutations) which ultimately facilitate the rational design of small molecule therapeutics [14].

The main aim of GEM models is to recapitulate genetic/molecular changes in human cancer and use these to test novel anticancer therapeutics in an attempt to accurately predict clinical response.

The first strains of genetically engineered mice predisposed to cancer were transgenic mouse models whereby cellular/viral oncogenes were introduced to the mouse germ line. One of the first transgenic cancer models involved the constitutive expression of the c-myc oncogene under the control of the mouse mammary tumor virus promoter leading to the development of mammary tumors. Many transgenic experiments have followed and clearly shown that the manipulation of the mouse germline could predispose the mice to cancer [15]. Upon the discovery that the progression to a malignant phenotype often involves the loss of TSG function, transgenic mouse models were developed which involved introducing a mutant TSG to the mouse germ line. TSG function can be impaired by either targeted gene knockout [16] or the transgenic expression of a dominant-negative form of the TSG. One of the first TSG mutants was the Rb "knockout" mouse [17]. Mice heterozygous for a null $\mathrm{Rb}$ allele developed tumors (pituitary adenocarcinomas, medullary thyroid carcinoma, and/or phaeochromocytomas). Since the $\mathrm{Rb}$ knockout, many mutant TSG or knockout cancer-prone mouse models have been developed including p53, Apc, Nf-1, and many more reviewed elsewhere. Alternatively, mouse models have been developed by inducing germline mutagenesis. For instance, the Min (multiple intestinal neoplasia) mouse was created by germline mutagenesis using $\mathrm{N}$-ethyl-N-nitrosourea, which causes a point mutation in the Apc TSG [18]. The most common human cancer, basal cell carcinoma, has also been modeled by the exposure of Ptchheterozygous mice to UV light [19]. Etiologic factors such as diet are also being modified and have been shown to affect tumor development in GEM models. Transgenic and knockout approaches can also be used to evaluate the role of specific components of the tumor microenvironment (e. g., matrix metalloproteinase 9) in tumor progression. The chemically induced skin carcinoma model (114) is another well-explored model that mimics more than one stage of tumor progression with defined genetic/molecular characteristics [H-ras activation, up-regulation of cyclin D1, loss of p53, and up-regulation of transforming growth factor-h1].

Transgenic and knockout mouse models involving manipulation of the mouse germline are often limited by embryonic lethality. In an attempt to overcome this, several new approaches to create GEM strains have been introduced, including transient conditional gene targeting, latent oncogenes, inducible oncogene expression and the use of avian sarcoma leucosis viruses, and chromosome engineering. These novel methods have been facilitated by recent technological advances [e.g., cytogenetic (spectral karyotyping), genomic comparative genomic hybridization, comparative genomic hybridization microarrays, and gene expression profiling Conditional transgenic/knockout models involve spatial control over the initiation of oncogene expression and TSGinactivation, respectively, and have been used to create models of several types of cancer [20]. The Cre-Lox system is the most widely used for both transient conditional knockout [21] and oncogene expression [22]. Another new approach in creating mouse models involves latent mutant alleles that become expressed following somatic recombination in vivo. Inducible oncogene expression involves the tissue-specific expression of oncogenes in response to stimulation by small molecules (e.g., doxycycline and tamoxifen;). Avian sarcoma leucosis viruses are also employed to deliver oncogenes and dominantnegative forms of TSGs to cells in vivo that express the avian sarcoma leucosis virus retroviral receptor.

Small molecule inhibitors have been used to target farnesyltransferase, epidermal growth factor receptor, and FLT3, usingGEM models and have been predominantly shown to block tumor development or regress established malignancy. RIP-Tag (pancreatic) and TRAMP (prostate) tumor progression models have been used to test the efficacy of angiogenesis/matrix metalloproteinase inhibitors and vascular endothelial growth factor receptor inhibitors, respectively. Additionally, very few studies have tested known clinically effective agents using GEM models Such GEM studies using mice (whereby the equivalent mutation found in human malignancy is validated and therapeutic efficacy is observed) provides optimism that GEM models may indeed be of value in predicting clinical response. Despite such promise, the value of GEM mouse models in anticancer drug discovery is yet to be determined. Only time will tell if GEM models will be any better at predicting clinical activity than currently used xenograft models.

Compared to xenograft models, relatively few studies have documented the use of orthotopic [23-25], transgenics and autochthonous models in cancer therapy and moreover in predicting clinical response. This is largely due to the fact that there are relatively few laboratories using these test systems in drug development programs. Additionally, unlike xenograft models, there are no studies collectively analyzing large bodies of data from these models, and therefore no definite conclusions can be made and the relative predictive value of orthotopic/autochthonous/transgenic models remains largely speculative. In order to assess the predictive value of $\backslash$ these models, preclinical studies testing currently used chemotherapeutic agents are required with time it is anticipated that the clinical use of small molecule therapeutics will outweigh the use of classic cytotoxics. As the identification of specific pathways driving the development of human cancer increases, it is imperative that transgenic models are developed that truly reflect clinical disease. New drug molecules will have been identified through a rational preclinical cascade culminating in the demonstration of in vivo "proof of principle" of efficacy, inappropriate preclinical mouse models. It is essential for the validation of transgenic models that such small molecule therapeutics are evaluated in patients that have been appropriately selected on the basis of the expression of the molecular target [26]

\section{Zebrafish models of human cancer}

\section{Zebrafish cancer models induced by chemicals}

While maintaining zebrafish in laboratory conditions, researchers observed diseases developing in adult fish, including cancer. Later studies clarified that after exposure of certain mutagens, zebrafish spontaneously developed almost any tumor type known from humans with similar morphology and comparable signalling pathways. The most common locations for this spontaneous neoplasia to arise include gut, thyroid, and liver. Lower levels of spontaneous neoplasia occur in blood vessels, brains, and gills. In light of spontaneous tumor acquisition, detailed chemical approaches to induce cancer have been developed [26] to chemically induce cancer, zebrafish are soaked in water dissolved with carcinogens for varied periods of time. Advantageously, zebrafish can endure treatments at a variety of chemical concentrations and durations. For instance, smaller doses, from $5 \mathrm{mmol}$ or less can be applied for up to $24 \mathrm{~h}$, while doses greater than $20 \mathrm{mmol}$ are to be applied for $8 \mathrm{~h}$ or less. The treatment of zebrafish with the mutagen 7,12-dimethylbenz(a) anthracene induces the broadest range of tumors, from epithelial tumors in intestines to mesenchymal tumors in blood vessels and lymphoid malignancies. Treatment with $\mathrm{N}$ nitrosodiethylamine is reported to induce pancreatic and liver carcinomas, while $\mathrm{N}$-nitrosodimethylamine specifically induces liver tumors

\section{Transgenic zebrafish cancer models}

Transgenic zebrafish expressing mammalian oncogenes provide a convenient platform for modeling human cancers through the misexpression of wild-type or constitutively active form of oncogenes under a zebrafish tissue-specific promoter. To generate transgenic zebrafish models, exogenous DNA is microinjected into one-cell-stage zebrafish embryos. Traditionally, linear or circular DNA plasmids, or artificial bacterial chromosomes are injected into fertilized zebrafish eggs. A large number of eggs need to be injected and screened to compensate for low germline transmission of the 
transgene of interest to the F1 generation. As transgenic lines are passed on through generations, repetitive DNA becomes susceptible to methylation, leading to the silencing of transgenes Modifications of these early transgenic techniques have led to the development of transposon-or I-SceImeganuclease-mediated transgenesis approaches that significantly improved germline transmission rates in zebrafish With these improved techniques, modeling human cancers in zebrafish through transgenesis becomes much easier. There are multiple types of cancers in zebrafish developed through the use of transgenesis MYC-induced T-cell Acute Lymphoblastic Leukemia (TALL) and melanoma models, in particular, have not only been used extensively to gain mechanistic insights into disease pathogenesis, but also in small molecule screens to successfully identify candidate therapeutics for human cancers.

\section{Zebrafish xenograft models of human cancer}

An additional methodology to establish cancer models involves the transplantation of human cancer cells into zebrafish embryos. Zebrafish lack an innate immune system until $72 \mathrm{~h}$ post-fertilization (hpf) and a mature adaptive immune response until $4 \mathrm{w}$ of life [27]. Therefore, human cancer cell lines, purified subpopulations of cancer cells or primary patient cells can be directly injected into zebrafish embryos to study many aspects of tumor biology, such as vasculature remodelling, cancer invasion, and metastasis. So far, multiple types of human cancer cell lines and primary patient samples, including gastrointestinal, neuroendocrine, leukemic, and melanoma clinical tumor samples have been successfully transplanted into 48-hpf zebrafish embryos and demonstrated their usefulness in studying cancer pathogenesis as well as novel drug screening and therapeutic testing of candidate cancer drugs. Invasiveness and micrometastasis of primary human tumors occur within $24 \mathrm{~h}$ of transplantation. These zebrafish xenograft models of human cancer are especially useful in drug screens allowing for the simultaneous examination of in vivo efficacy and toxicity of candidate drugs. Finally, the advent of pigmentlessCasper adult fish has enabled visualization of tumor cell proliferation and dissemination in transplanted recipients beyond zebrafish embryonic stages. Adult zebrafish have three distinct classes of pigment cells: black melanophores, reflective iridophores, and yellow xanothophores. Nacre mutant zebrafish lack melanocytes, while royorbison zebrafish lack iridophores. Casperzebrafish are double mutant for nacre and roy lacking both melanocytes and iridophores throughout embryogenesis and adulthood. Casper permits all organs to be seen with a stereomicroscope.

\section{CONCLUSION}

Here in this review discovered more about the underlying mechanisms responsible for the initiation and progression of human cancers, also experienced a move away from the development of classic cytotoxic agents to the rational design of small molecule anticancer therapeutics. This has prompted a transition from empirical compound-orientated preclinical screening to target-orientated drug screening. The use of uncharacterized tumor models (s. c. xenografts/syngeneic models) has been continuously replaced by more clinically relevant and molecularly characterized models along with the integration of pharmacodynamic and pharmacokinetic approaches. The value of any preclinical tumor model will ultimately depend on its ability to accurately predict clinical response. In this modern era of target-driven anticancer drug discovery, we believe that the full potential of any tumor model can only be met if it is used "appropriately," that is, if it is fully characterized to ensure that the molecular target of interest is expressed and that the model is used to confirm drug-target interaction. In addition to determining quantitative antitumor activity, it is believed that preclinical tumor models should use be used to gain a broad range of be used to gain information (i.e., pharmacokinetics/metabolism/pharmacodynamics). It is emphasized that this preclinical information needs to be used appropriately in clinical trials. In particular, if a novel target directed agent is to be used to treat a patient, the presence of its respective target must initially be confirmed. If preclinical models are used routinely to this extent and a closer relationship exists between the clinician and the laboratory scientist, it is anticipated that the clinical use of small molecule.

\section{AUTHORS CONTRIBUTIONS}

All the author have contributed equally

\section{CONFLICT OF INTERESTS}

\section{Declare none}

\section{REFERENCES}

1. Goldin A, Venditti JM, Kline I, Mantel N. Evaluation of antileukemic agents employing advanced leukemia L1210 in mice. Cancer Res 1959;19:429-66.

2. Staquet MJ, Byar DP, Green SB, Rozencweig M. Clinical predictivity of transplantable tumor systems in the selection of new drugs for solid tumors: rationale for a three-stage strategy. Cancer Treat Rep 1983;67:753-65.

3. Shoemaker RH, Wolpert-DeFilippes MK, Kern DH. Application of a human tumor colony-forming assay to new drug screening. Cancer Res 1985;45:2145-53.

4. Von Hoff DD, Clark GM, Stogdill BJ. Prospective clinical trial of a human tumor cloning system. Cancer Res 1983;43:1926-31.

5. Qiuwen Mi, John M Pezzuto. Use of the in vivo hollow fiber assay in natural products anticancer drug discovery. J Nat Prod 2009;72:573-80.

6. Kerbel RS. Human tumor xenografts as predictive preclinical models for anticancer drug activity in humans: better than commonly perceived but they can be improved. Cancer Biol Ther 2003;2:S134-9.

7. Paget S. Secondary growths of cancer of the breast. Lancet 1889;1:571-3.

8. Fidler IJ. Rationale and methods for the use of nude mice to study the biology and therapy of human cancer metastasis. Cancer Metastasis Rev 1986;5:29-49.

9. Fidler IJ, Naito S, Pathak S. Orthotopic implantation is essential for the selection, growth and metastasis of human renal cell cancer in nude mice [corrected]. Cancer Metastasis Rev 1990;9:149-65.

10. Hoffman RM. Fertile seed and rich soil: the development of clinically relevant models of human cancer by surgical orthotopic implantation of intact tissues. In: Teicher B. editor. Anticancer drug development guide: preclinical screening, clinical trials, and approval. Totowa NJ: Humana Press: Inc; 1997. p. 127-44.

11. Hoffman RM. Visualisation of metastasis in orthotopic mouse models with green fluorescent protein. In: Fiebig HH, Burger AM. editors. Relevance of tumour models for anticancer drug development. Contrib Oncol. Vol. 54. Basel: Karger; 1999. p. 81-7.

12. Kruger AE, Duray PH, Douglas K. Approaches to a preclinical screening of antiangiogenic agents. Semin Oncol 2001;28:570-6.

13. Berger MR. Autochthonous tumour models in rats: Is there a relevance for anticancer drug development. In: Fiebig $\mathrm{HH}$, Burger AM. Editors. Relevance of Tumour Models for Anticancer Drug Development. Contrib Oncol. Vol. 54. Basel: Karger; 1999. p. 15-27.

14. Macleod KF, Jacks T. Insights into cancer from transgenic mouse models. J Pathol 1999;187:43-60.

15. Adams JM, Cory S. Transgenic models for haemopoietic malignancies. Biochim Biophys Acta 1991;1072:9-31.

16. Lavigueur A, Maltby V, Mock D. High incidence of lung, bone, and lymphoid tumors in transgenic mice overexpressing mutant alleles of the p53 oncogene. Mol Cell Biol 1989;9:3982-91.

17. Jacks T, Fazeli A, Schmitt EM. Effects of an Rb mutation in the mouse. Nature 1992;359:295-300.

18. Shoemaker AR, Gould KA, Luongo C, Moser AR, Dove WF. Studies of neoplasia in the min mouse. Biochim Biophys Acta 1997;1332:F25-48.

19. Aszterbaum M, Epstein J, Oro A. Ultraviolet and ionizing radiation enhance the growth of BCCs and trichoblastomas in patched heterozygous knockout mice. Nat Med 1999;5:1285-91.

20. Pinkel D, Segraves R, Sudar D. High-resolution analysis of DNA copy number variation using comparative genomic hybridization to microarrays. Nat Genet 1998;20:207-11.

21. Le Y, Sauer B. Conditional gene knockout using Cre recombinase. Mol Biotechnol 2001;17:269-75. 
22. Lakso M, Sauer B, Mosinger B Jr. Targeted oncogene activation by site-specific recombination in transgenic mice. Proc Natl Acad Sci USA 1992:89:6232-6.

23. Kuo TH, Kubota T, Watanabe M. Site-specific chemosensitivity of human small-cell lung carcinoma growing orthotopically compared to subcutaneously in SCID mice: the importance of orthotopic models to obtain relevant drug evaluation data. Anticancer Res 1993;13:627-30.

24. Fidler IJ, Ellis LM. The implications of angiogenesis for the biology and therapy of cancer metastasis. Cell 1994;79:185-8.
25. Cowen SE, Bibby MC, Double JA. Characterisation of the vasculature within a murine adenocarcinoma growing in different sites to evaluate the potential of vascular therapies. Acta Oncol 1995;34:357-60.

26. Marie Suggitt, Michael C Bibby. 50 y of preclinical anticancer drug screening: empirical to target-driven approaches. Clin Cancer Res 2005;11:971-81.

27. JF Shepard, JL Stern, HM, Zon LI. Zebrafish as a cancer model system. Cancer Cell 2002;1:229-31. 